\title{
Acoustical studies on molecular interaction of 1,3,4-pyrazoline derivatives using ultrasonic technique at $303.15 \mathrm{~K}$
}

\author{
A. Raguraman, N. Santhi ${ }^{*}$ \\ Department of Chemistry, Government Arts College, Chidambaram - 608201, Tamil Nadu, India \\ *E-mail address: nsaanthi@gmail.com
}

\begin{abstract}
The acoustical parameters for the binary liquid mixtures containing Pyrazoline derivatives in DMF have been estimated at $303.15 \mathrm{~K}$, from the measured values of ultrasonic velocity (U), density $(\rho)$ and viscosity $(\eta)$. From these data some of acoustical parameters such as adiabatic compressibility $(\beta)$, free length $\left(\mathrm{L}_{\mathrm{f}}\right)$, acoustic impedance $(\mathrm{Z})$, Rao's constant $(\mathrm{R})$, molar compressibility $(\mathrm{W})$, relaxation time $(\tau)$, free volume $\left(\mathrm{V}_{\mathrm{f}}\right)$ and internal pressure $\left(\pi_{\mathrm{i}}\right)$, etc., have been computed using the standard relations. The results have been discussed in terms of molecular interactions.
\end{abstract}

Keywords: Binary mixture; molecular interaction; ultrasonic velocity; density; viscosity; free length

\section{INTRODUCTION}

The study of ultrasonics in organic liquids has been the subject of extensive research recently. Ultrasonic methods have the added advantage of being less cost with efficiency comparable to other methods. Hence, a number of works have reported the study through ultrasonic method [1-4]. Acoustics is a field widely used in recent years to study various molecular interactions. The study of the liquid state properties using spectroscopic and acoustical methods provides valuable information for their varied usage.

The study of liquid mixtures containing of polar and non-polar components find applications in industrial and technological process [5]. The mixing of different components gives rise to solutions that generally do not behave ideally [6-7]. Further these properties have been widely used to study the molecular interaction between the various species in the mixture [8-9]. In recent years, the measurement of ultrasonic velocity has been extensively applied in understanding the nature of molecular systems, physicochemical behaviour and molecular interactions in liquid mixtures [10-12].

Intermolecular interaction in various binary liquid mixtures at different temperatures have been studied by several authors[13-16]. Ultrasonic velocity and related thermodynamic parameters helps us for characterizing thermodynamic and physico-chemical aspects of binary liquid mixtures such as molecular association and dissociation [17-18]. Viscosity, density measurements and the properties derived from these are excellent tools to detect solute solute and solute - solvent interactions. 
Physico-chemical properties like density, viscosity and speed of sound have got considerable importance in forming theoretical models as well as their applications in a number of branches of science. A considerable progress has been made theoretical understanding of liquid-liquid mixture [19-22].

\section{EXPERIMENTAL}

\section{1. Choice of Solvent}

Dimethyl sulphoxide is chosen as solvent in the present work. This solvent is of industrial interest because of their wide use as solvent and solubilizing agent.

The densities, viscosities and ultrasonic velocities of solvent and solutions of different concentrations were measured at $303.15 \mathrm{~K}$ by specific gravity bottle, an Ostwald's viscometer and single frequency ultrasonic interferometer operating at $2 \mathrm{MHz}$.

\section{2. Density}

The density of pure liquids and mixtures are measured using a $10 \mathrm{ml}$ specific gravity bottle. The measured density was calculated using the formula

$$
\rho_{2}=\left(\mathrm{w}_{2} / \mathrm{w}_{1}\right) \rho_{1}
$$

where:

$\mathrm{w}_{1}$ is the weight of the distilled water

$\mathrm{w}_{2}$ is the weight of the experimental liquid

$\rho_{1}$ is the density of water

\section{3. Viscosity}

The viscosity of the pure liquids and liquid mixtures are measured using an Ostwald's Viscometer calibrated with doubly distilled water. The Ostwald's Viscometer with the experimental liquid is immersed in a temperature controlled water bath at $303.15 \mathrm{~K}$. The digital stopwatch, with an accuracy of $\pm 0.01 \mathrm{sec}$ was used to determine flow time of solutions. Using the flow times $(\mathrm{t})$ and known of standard water sample, the viscosity of solvent and solutions were determined according the following equation:

$$
\eta_{2}=\eta_{1}\left(t_{2} / t_{1}\right)\left(\rho_{2} / \rho_{1}\right)
$$

where:

$\eta_{1}$ is the viscosity of water

$t_{1}$ is the time of flow of water

$\rho_{1}$ is the density of water

$\eta_{2}$ is the viscosity of the binary mixture

$t_{2}$ is the time of flow of the binary mixture

$\rho_{2}$ is the density of the binary mixture 


\section{4. Ultrasonic velocity}

The sound velocity of the liquid mixture have been measured using an ultrasonic interferometer (Mittal Enterprises, New Delhi) working at a fixed frequency of $2 \mathrm{MH}_{\mathrm{Z}}$. The binary liquid mixture is filled in the measuring cell with quartz crystal and then micrometer was fixed. The circulation of water from the thermostat at $303.15 \mathrm{~K}$ was started and test solution in the cell is allowed to thermally equilibrate. The micrometer was rotated very slowly so as to obtain a maximum or minimum of anode current (n). A number of maximum reading of anode current were counted. The total distance (d) travel by the micrometer for $\mathrm{n}=$ 10, was read. The wave length $(\lambda)$ was determined according to the following equation:

$$
\lambda=2 \mathrm{~d} / \mathrm{n}
$$

The sound velocity (U) of solvent and solutions were calculated from the wavelength and frequency $(\mathrm{F})$ according to the following equation:

$$
\mathrm{U}=\lambda \mathrm{F}
$$

\section{THEORY}

The ultrasonic velocity $(\mathrm{U})$, density $(\rho)$ and viscosity $(\eta)$, molar volume $(\mathrm{Vm})$ in pure liquids and liquid mixtures of various concentrations have been measured at $303.15 \mathrm{~K}$. Thermodynamic and acoustical parameters such as adiabatic compressibility $(\beta)$, free length $\left(\mathrm{L}_{\mathrm{f}}\right)$, acoustic impedance $(\mathrm{Z})$, relative association $\left(\mathrm{R}_{\mathrm{A}}\right)$, relaxation strength $(\mathrm{r})$, Rao's constant $(\mathrm{R})$, molar compressibility $(\mathrm{W})$, relaxation time $(\tau)$, Vander waal's constant $(\mathrm{b})$, Isothermal compressibility $\left(\beta_{\mathrm{T}}\right)$, Isothermal expansion coefficient $(\alpha)$, ultrasonic attenuation $\left(\alpha / \mathrm{f}^{2}\right)$, free volume $\left(\mathrm{V}_{\mathrm{f}}\right)$ and internal pressure $\left(\pi_{\mathrm{i}}\right)$ were determined using the observed values of velocity, density $\&$ viscosity using the standard relations given below.

1. The adiabatic compressibility $(\beta)$ has been calculated from sound velocity $(\mathrm{U})$ and the density $(\rho)$ of the medium using the relation

$$
\beta=1 / \mathrm{U}^{2} \rho
$$

2. Intermolecular free length $\left(\mathrm{L}_{\mathrm{f}}\right)$ is calculated using the standard expression

$$
\mathrm{L}_{\mathrm{f}}=\mathrm{K} \beta^{1 / 2}
$$

where $\mathrm{K}$ is a Jacobson's constant $\left(=2.0965 \mathrm{X} 10^{-6}\right)$

3. Acoustic impedance $(Z)$ was calculated by the equation

$$
\mathrm{Z}=\mathrm{U} \rho
$$

where $\rho$ is the density of the mixture and $U$ is the ultrasonic velocity of the mixture.

4. Molar compressibility or Wada's constant (W) can be calculated by the following equation

$$
\mathrm{W}=(\mathrm{M} / \rho) \beta^{-1 / 7}
$$

where $\mathrm{M}$ is the molecular weight of the solution which can be calculated according to 
the equation

$$
\mathrm{M}=\mathrm{M}_{1} \mathrm{X}_{1}+\mathrm{M}_{2} \mathrm{X}_{2}
$$

where $X_{1}$ and $X_{2}$ are mole fractions of solvent and solute, respectively. $M_{1}$ and $M_{2}$ are the molecular weights of the solvent and solute respectively.

5. The molar sound velocity or Rao's constant (R) was calculated by the equation

$$
\mathrm{R}=(\mathrm{M} / \rho) \mathrm{U}^{1 / 3}
$$

where $\rho$ is the density and $U$ is the ultrasonic velocity of the mixture.

6. The Relative association $\left(\mathrm{R}_{\mathrm{A}}\right)$ was calculated by the following equation

$$
R_{\mathrm{A}}=\rho / \rho_{\mathrm{o}}\left(\mathrm{U}_{\mathrm{o}} / \mathrm{U}\right)^{1 / 3}
$$

where $U, U_{o}$ and $\rho, \rho_{o}$ are ultrasonic velocities and densities of solution and solvent respectively.

7. Relaxation strength (r) was calculated by the following equation

$$
\mathrm{r}=1-\left[\mathrm{U} / \mathrm{U}_{\infty}\right]^{2}
$$

where $U_{\infty}=1600 \mathrm{~m} / \mathrm{sec}$.

8. Vander Waals constant (b) was calculated by the following equation

$$
\mathrm{b}=(\mathrm{M} / \rho)\left[1-\left(\mathrm{RT} / \mathrm{MU}^{2}\right)\left\{\left(1+\mathrm{MU}^{2} / 3 \mathrm{RT}\right)^{1 / 2}-1\right\}\right]
$$

where $\mathrm{R}$ is the gas constant $\left(=8.314 \mathrm{JK}^{-1} \mathrm{~mol}^{-1}\right)$ and $\mathrm{T}$ is the absolute temperature.

9. Viscous relaxation time $(\tau)$ was calculated by the following equation

$$
\tau=4 \eta / 3 \rho U^{2}
$$

where $\eta$ is the viscosity, $\rho$ is the density and $U$ is the ultrasonic velocity of the mixture.

10. Isothermal compressibility, $\beta_{\mathrm{T}}$

$$
\beta_{\mathrm{T}}=1.71 \times 10^{-3} / \mathrm{T}^{4 / 9} \mathrm{U}^{2} \rho^{4 / 3}
$$

where, $\mathrm{U}$ is the ultrasonic velocity, $\rho$ is the density and $\mathrm{T}$ is the Temperature.

11. Isothermal Expansion coefficient, $\alpha$

$$
\alpha=\left(0.0191 \beta_{\mathrm{T}}\right)^{1 / 4}
$$

12. Ultrasonic attenuation $\left(\alpha / \mathrm{f}^{2}\right)$

$$
\left(\alpha / f^{2}\right)=8 \pi^{2} \eta / 3 \rho U^{2}
$$

13. Free volume $\left(\mathrm{V}_{\mathrm{f}}\right)$ was calculated by the following equation

$$
\mathrm{V}_{\mathrm{f}}=[\mathrm{MU} / \mathrm{K \eta}]^{3 / 2}
$$

where $\mathrm{K}$ is a constant $\left(=4.28 \times 10^{9}\right)$ 
14. The Internal pressure $\left(\pi_{\mathrm{i}}\right)$ was calculated by the following equation

$$
\pi_{\mathrm{i}}=\mathrm{bRT}[\mathrm{K} \eta / \mathrm{U}]^{1 / 2} \rho^{2 / 3} / \mathrm{M}^{7 / 6}
$$

where $\mathrm{b}$ is the packing factor $(=2), \mathrm{K}$ is a constant $\left(=4.28 \mathrm{X} \mathrm{10^{9 }}\right)$

The experimental density $(\rho)$, viscosity $(\eta)$ ultrasonic velocity (U) for Pyrazoline derivatives at various concentrations are given in Tables (1-3). The computed acoustical parameters for the above Pyrazoline derivatives are given in Tables (4-12).

Table 1. Ultrasonic velocity (U), Density $(\rho)$, Viscosity $(\eta)$ and Molar volume (Vm) of Pyrazoline derivatives with DMF at $303.15 \mathrm{~K}$.

\begin{tabular}{|c|c|c|c|c|}
\hline \multirow{2}{*}{ Concentration } & \multicolumn{4}{|c|}{ Pyrazoline derivatives (P1-P10) } \\
\hline & $\mathrm{U}$ & $\rho$ & $\eta$ & $\mathrm{Vm}$ \\
\hline (M) & $(\mathrm{m} / \mathrm{s})$ & $\left(\mathrm{Kg} / \mathrm{m}^{3}\right)$ & $(\mathrm{mPa} \cdot \mathrm{s})$ & $\left(10^{-6} \mathrm{~m}^{3} / \mathrm{mole}\right)$ \\
\hline \multicolumn{5}{|c|}{ Compound P1 } \\
\hline 0.02 & 1457 & 961.7 & 0.9288 & 83.98 \\
\hline 0.04 & 1470 & 978.2 & 0.9517 & 90.41 \\
\hline 0.06 & 1480 & 997.2 & 0.9702 & 96.39 \\
\hline 0.08 & 1490 & 1012.5 & 0.9923 & 102.52 \\
\hline 0.1 & 1496 & 1032.8 & 1.017 & 107.94 \\
\hline \multicolumn{5}{|c|}{ Compound P2 } \\
\hline 0.02 & 1487 & 961.4 & 0.956 & 84.09 \\
\hline 0.04 & 1493 & 978 & 0.9725 & 90.59 \\
\hline 0.06 & 1495 & 997.1 & 0.9917 & 96.64 \\
\hline 0.08 & 1508 & 1000.3 & 1.009 & 104.09 \\
\hline 0.1 & 1512 & 1010.8 & 1.0135 & 110.68 \\
\hline \multicolumn{5}{|c|}{ Compound P3 } \\
\hline 0.02 & 1437 & 961.8 & 0.9771 & 85.09 \\
\hline 0.04 & 1440 & 978.3 & 0.9938 & 92.61 \\
\hline 0.06 & 1447 & 980.7 & 1.0033 & 101.32 \\
\hline 0.08 & 1509 & 996 & 1.0332 & 108.55 \\
\hline 0.1 & 1511 & 1016.3 & 1.0542 & 115.01 \\
\hline
\end{tabular}

Table 2. Ultrasonic velocity (U), Density ( $\rho$ ), Viscosity $(\eta)$ and Molar volume (Vm) of Pyrazoline derivatives with DMF at $303.15 \mathrm{~K}$.

\begin{tabular}{|c|c|c|c|c|}
\hline \multirow{2}{*}{ Concentration } & \multicolumn{4}{|c|}{ Pyrazoline derivatives (P1-P10) } \\
\cline { 2 - 5 } & $\mathrm{U}$ & $\rho$ & $\eta$ & $\mathrm{Vm}$ \\
\hline$(\mathrm{M})$ & $(\mathrm{m} / \mathrm{s})$ & $\left(\mathrm{Kg} / \mathrm{m}^{3}\right)$ & $(\mathrm{mPa} \cdot \mathrm{s})$ & $\left(10^{-6} \mathrm{~m}^{3} / \mathrm{mole}\right)$ \\
\hline
\end{tabular}




\begin{tabular}{|c|c|c|c|c|}
\hline \multicolumn{5}{|c|}{ Compound P4 } \\
\hline 0.02 & 1464 & 961.3 & 0.9697 & 84.62 \\
\hline 0.04 & 1469 & 979.1 & 0.9876 & 91.51 \\
\hline 0.06 & 1475 & 980.6 & 0.9962 & 99.80 \\
\hline 0.08 & 1498 & 995.6 & 1.0127 & 106.59 \\
\hline 0.1 & 1504 & 1015.7 & 1.0391 & 112.61 \\
\hline \multicolumn{5}{|c|}{ Compound P5 } \\
\hline 0.02 & 1434 & 962 & 0.9222 & 83.79 \\
\hline 0.04 & 1457 & 981.7 & 0.9411 & 89.76 \\
\hline 0.06 & 1474 & 1000.8 & 0.9737 & 95.56 \\
\hline 0.08 & 1486 & 1019.3 & 0.999 & 101.20 \\
\hline 0.1 & 1515 & 1037.2 & 1.0166 & 106.71 \\
\hline \multicolumn{7}{|c|}{ Compound P6 } \\
\hline 0.02 & 1406 & 963.4 & 0.9235 & 83.27 \\
\hline 0.04 & 1464 & 981.4 & 0.9478 & 89.02 \\
\hline 0.06 & 1478 & 1000.4 & 0.9733 & 94.46 \\
\hline 0.08 & 1481 & 1018.8 & 0.9912 & 99.76 \\
\hline 0.1 & 1517 & 1037 & 1.0238 & 104.89 \\
\hline
\end{tabular}

Table 3. Ultrasonic velocity (U), Density ( $\rho$ ), Viscosity $(\eta)$ and Molar volume (Vm) of Pyrazoline derivatives with DMF at $303.15 \mathrm{~K}$.

\begin{tabular}{|c|c|c|c|c|}
\hline \multirow{2}{*}{ Concentration } & \multicolumn{5}{|c|}{ Pyrazoline derivatives (P1-P10) } \\
\cline { 2 - 5 } & $\mathrm{U}$ & $\rho$ & $\eta$ & Vm \\
\hline \multirow{2}{*}{$(\mathrm{M})$} & $(\mathrm{m} / \mathrm{s})$ & $\left(\mathrm{Kg} / \mathrm{m}^{3}\right)$ & $(\mathrm{mPa} \cdot \mathrm{s})$ & $\left(10^{-6} \mathrm{~m}^{3} / \mathrm{mole}\right)$ \\
\hline \multicolumn{5}{|c|}{ Compound P7 } \\
\hline 0.02 & 1430 & 963.1 & 0.9646 & 84.00 \\
\hline 0.04 & 1452 & 980.8 & 0.9823 & 90.46 \\
\hline 0.06 & 1472 & 999.9 & 1.0086 & 96.55 \\
\hline 0.08 & 1486 & 1019.8 & 1.0433 & 102.33 \\
\hline 0.1 & 1512 & 1037.9 & 1.0692 & 108.08 \\
\hline \multicolumn{5}{|c|}{ Compound P8 } \\
\hline 0.02 & 1413 & 961.2 & 0.9367 & 83.84 \\
\hline 0.04 & 1414 & 976.1 & 0.9457 & 90.24 \\
\hline 0.06 & 1430 & 995.2 & 0.9836 & 96.04 \\
\hline 0.08 & 1435 & 1000.3 & 0.994 & 103.05 \\
\hline 0.1 & 1446 & 1019.2 & 1.0099 & 108.49 \\
\hline \multicolumn{5}{|c|}{ Compound P9 } \\
\hline
\end{tabular}




\begin{tabular}{|c|c|c|c|c|}
\hline 0.02 & 1403 & 962.7 & 0.9345 & 83.62 \\
\hline 0.04 & 1420 & 975.1 & 0.9508 & 90.17 \\
\hline 0.06 & 1446 & 989.1 & 0.9672 & 96.39 \\
\hline 0.08 & 1469 & 999.6 & 0.9802 & 102.80 \\
\hline 0.1 & 1480 & 1008.3 & 1.0014 & 109.27 \\
\hline \multicolumn{5}{|c|}{ Compound P10 } \\
\hline 0.02 & 1448 & 967.6 & 0.9494 & 83.53 \\
\hline 0.04 & 1473 & 984.5 & 0.9727 & 89.96 \\
\hline 0.06 & 1476 & 994.1 & 0.9816 & 96.87 \\
\hline 0.08 & 1477 & 1007.5 & 0.9958 & 103.26 \\
\hline 0.1 & 1486 & 1014.3 & 1.0121 & 110.20 \\
\hline
\end{tabular}

Table 4. Specific Acoustic Impedance $(Z)$, Adiabatic compressibility $\left(\beta_{\mathrm{ad}}\right)$, Intermolecular Free Length $\left(\mathrm{L}_{\mathrm{f}}\right)$, Rao's Constant $(\mathrm{R})$ and Molar compressibility $(\mathrm{W})$ of Pyrazoline derivatives with DMF at $303.15 \mathrm{~K}$.

\begin{tabular}{|c|c|c|c|c|c|}
\hline \multirow{2}{*}{ Concentration } & \multicolumn{5}{|c|}{ Pyrazoline derivatives (P1-P10) } \\
\cline { 2 - 6 } & $\mathrm{Z}$ & $\beta_{\mathrm{ad}}$ & $\mathrm{L}_{\mathrm{f}}$ & $\mathrm{R}$ & $\mathrm{W}$ \\
\hline \multirow{2}{*}{$(\mathrm{M})$} & $\left(10^{3} \mathrm{Kg} / \mathrm{m}^{2} \mathrm{~s}\right)$ & $\left(10^{-10} \mathrm{~Pa}^{-1}\right)$ & $\left(10^{-10} \mathrm{~m}\right)$ & $\begin{array}{c}\left(\mathrm{m}^{3} / \mathrm{mole}\right) \\
(\mathrm{m} / \mathrm{s})^{1 / 3}\end{array}$ & $\begin{array}{c}\left(\mathrm{m}^{3} / \mathrm{mole}\right)( \\
\left.\mathrm{N} / \mathrm{m}^{2}\right)^{1 / 7}\end{array}$ \\
\hline 0.02 & 1401.197 & 4.8983 & 0.4639 & 0.9521 & 1.7955 \\
\hline 0.04 & 1437.954 & 4.7308 & 0.4559 & 1.0280 & 1.9426 \\
\hline 0.06 & 1475.856 & 4.5782 & 0.4485 & 1.0985 & 2.0808 \\
\hline 0.08 & 1508.625 & 4.4487 & 0.4421 & 1.1709 & 2.2221 \\
\hline 0.1 & 1545.069 & 4.3263 & 0.4360 & 1.2345 & 2.3489 \\
\hline \multicolumn{5}{|c|}{ Compound P1 } \\
\hline 0.02 & 1429.602 & 4.7041 & 0.4547 & 0.9598 & 1.8083 \\
\hline 0.04 & 1460.154 & 4.5871 & 0.4490 & 1.0354 & 1.9552 \\
\hline 0.06 & 1490.665 & 4.4872 & 0.4441 & 1.1050 & 2.0922 \\
\hline 0.08 & 1508.452 & 4.3961 & 0.4395 & 1.1936 & 2.260 \\
\hline 0.1 & 1528.33 & 4.3274 & 0.4361 & 1.2704 & 2.4086 \\
\hline \multicolumn{7}{|c|}{ Compound P3 } \\
\hline 0.02 & 1382.107 & 5.035 & 0.4704 & 0.9603 & 1.8122 \\
\hline 0.04 & 1408.752 & 4.9295 & 0.4654 & 1.0458 & 1.9782 \\
\hline 0.06 & 1419.073 & 4.87 & 0.4626 & 1.1460 & 2.1679 \\
\hline 0.08 & 1502.964 & 4.4092 & 0.4402 & 1.2451 & 2.3560 \\
\hline 0.1 & 1535.629 & 4.3097 & 0.4352 & 1.3197 & 2.5041 \\
\hline
\end{tabular}


Table 5. Specific Acoustic Impedance $(Z)$, Adiabatic compressibility $\left(\beta_{\mathrm{ad}}\right)$, Intermolecular Free Length $\left(\mathrm{L}_{\mathrm{f}}\right)$, Rao's Constant $(\mathrm{R})$ and Molar compressibility $(\mathrm{W})$ of Pyrazoline derivatives with

DMF at $303.15 \mathrm{~K}$.

\begin{tabular}{|c|c|c|c|c|c|}
\hline \multirow{2}{*}{ Concentration } & \multicolumn{5}{|c|}{ Pyrazoline derivatives (P1-P10) } \\
\hline & $\mathrm{Z}$ & $\beta_{\mathrm{ad}}$ & $\mathrm{L}_{\mathrm{f}}$ & $\mathrm{R}$ & W \\
\hline$(\mathrm{M})$ & $\left(10^{3} \mathrm{Kg} / \mathrm{m}^{2} \mathrm{~s}\right)$ & $\left(10^{-10} \mathrm{~Pa}^{-1}\right)$ & $\left(10^{-10} \mathrm{~m}\right)$ & $\begin{array}{c}\left(\mathrm{m}^{3} / \text { mole }\right) \\
(\mathrm{m} / \mathrm{s})^{1 / 3}\end{array}$ & $\begin{array}{l}\left(\mathrm{m}^{3} / \text { mole }\right) \\
\left(\mathrm{N} / \mathrm{m}^{2}\right)^{1 / 7}\end{array}$ \\
\hline \multicolumn{6}{|c|}{ Compound P4 } \\
\hline 0.02 & 1407.343 & 4.8535 & 0.4618 & 0.9608 & 1.8115 \\
\hline 0.04 & 1438.298 & 4.7329 & 0.4560 & 1.0403 & 1.9662 \\
\hline 0.06 & 1446.385 & 4.6873 & 0.4538 & 1.1360 & 2.1471 \\
\hline 0.08 & 1491.409 & 4.476 & 0.4435 & 1.2196 & 2.3084 \\
\hline 0.1 & 1527.613 & 4.3525 & 0.4373 & 1.2902 & 2.4485 \\
\hline \multicolumn{6}{|c|}{ Compound P5 } \\
\hline 0.02 & 1379.508 & 5.0551 & 0.4713 & 0.9449 & 1.7833 \\
\hline 0.04 & 1430.337 & 4.7985 & 0.4592 & 1.0176 & 1.9248 \\
\hline 0.06 & 1475.179 & 4.5989 & 0.4495 & 1.0876 & 2.0616 \\
\hline 0.08 & 1514.68 & 4.4428 & 0.4419 & 1.1549 & 2.1941 \\
\hline 0.1 & 1571.358 & 4.2006 & 0.4296 & 1.2255 & 2.3320 \\
\hline \multicolumn{6}{|c|}{ Compound P6 } \\
\hline 0.02 & 1354.54 & 5.2508 & 0.4804 & 0.9329 & 1.7628 \\
\hline 0.04 & 1436.77 & 4.7541 & 0.4571 & 1.0108 & 1.9113 \\
\hline 0.06 & 1478.591 & 4.5759 & 0.4484 & 1.0760 & 2.0393 \\
\hline 0.08 & 1508.843 & 4.4751 & 0.4435 & 1.1372 & 2.1606 \\
\hline 0.1 & 1573.129 & 4.1903 & 0.4291 & 1.2053 & 2.2932 \\
\hline
\end{tabular}

Table 6. Specific Acoustic Impedance $(Z)$, Adiabatic compressibility $\left(\beta_{\mathrm{ad}}\right)$, Intermolecular Free Length $\left(\mathrm{L}_{\mathrm{f}}\right)$, Rao's Constant $(\mathrm{R})$ and Molar compressibility $(\mathrm{W})$ of Pyrazoline derivatives with DMF at $303.15 \mathrm{~K}$.

\begin{tabular}{|c|c|c|c|c|c|}
\hline \multirow{2}{*}{ Concentration } & \multicolumn{5}{|c|}{ Pyrazoline derivatives (P1-P10) } \\
\cline { 2 - 6 } & $\mathrm{Z}$ & $\beta_{\mathrm{ad}}$ & $\mathrm{L}_{\mathrm{f}}$ & $\mathrm{R}$ & $\mathrm{W}$ \\
\hline \multirow{2}{*}{$(\mathrm{M})$} & $\left(10^{3} \mathrm{Kg} / \mathrm{m}^{2} \mathrm{~s}\right)$ & $\left(10^{-10} \mathrm{~Pa}^{-1}\right)$ & $\left(10^{-10} \mathrm{~m}\right)$ & $\begin{array}{c}\left(\mathrm{m}^{3} / \mathrm{mole}\right) \\
(\mathrm{m} / \mathrm{s})^{1 / 3}\end{array}$ & $\begin{array}{c}\left(\mathrm{m}^{3} / \mathrm{mole}^{2}\right) \\
\left(\mathrm{N} / \mathrm{m}^{2}\right)^{1 / 7}\end{array}$ \\
\hline \multicolumn{7}{|c|}{ Compound P7 } \\
\hline 0.02 & 1377.233 & 5.0776 & 0.4724 & 0.9464 & 1.7868 \\
\hline 0.04 & 1424.122 & 4.836 & 0.4610 & 1.0243 & 1.9375 \\
\hline 0.06 & 1471.853 & 4.6156 & 0.4504 & 1.0983 & 2.0818 \\
\hline 0.08 & 1515.423 & 4.4407 & 0.4417 & 1.1678 & 2.2187 \\
\hline 0.1 & 1569.305 & 4.2144 & 0.4303 & 1.2405 & 2.3609 \\
\hline 0.02 & 1358.176 & 5.2108 & 0.4785 & 0.9408 & 1.7767 \\
\hline 0.04 & 1380.205 & 5.124 & 0.4745 & 1.0128 & 1.9169 \\
\hline
\end{tabular}




\begin{tabular}{|c|c|c|c|c|c|}
\hline 0.06 & 1423.136 & 4.9138 & 0.4647 & 1.0820 & 2.0524 \\
\hline 0.08 & 1435.431 & 4.8547 & 0.4619 & 1.1623 & 2.2059 \\
\hline 0.1 & 1473.763 & 4.6925 & 0.4541 & 1.2269 & 2.3338 \\
\hline \multicolumn{7}{|c|}{ Compound P9 } \\
\hline 0.02 & 1350.668 & 5.2771 & 0.4816 & 0.9361 & 1.7689 \\
\hline 0.04 & 1384.642 & 5.086 & 0.4728 & 1.0135 & 1.9174 \\
\hline 0.06 & 1430.239 & 4.8353 & 0.4610 & 1.0900 & 2.0646 \\
\hline 0.08 & 1468.412 & 4.6359 & 0.4513 & 1.1686 & 2.2152 \\
\hline 0.1 & 1492.284 & 4.5278 & 0.4461 & 1.2452 & 2.3626 \\
\hline \multicolumn{7}{|c|}{ Compound P10 } \\
\hline 0.02 & 1401.085 & 4.9291 & 0.4654 & 0.9450 & 1.7843 \\
\hline 0.04 & 1450.169 & 4.6814 & 0.4536 & 1.0235 & 1.9357 \\
\hline 0.06 & 1467.292 & 4.6174 & 0.4504 & 1.1030 & 2.0886 \\
\hline 0.08 & 1488.078 & 4.5498 & 0.4471 & 1.1760 & 2.2312 \\
\hline 0.1 & 1507.25 & 4.4647 & 0.4429 & 1.2575 & 2.3875 \\
\hline
\end{tabular}

Table 7. Relaxation time $(\tau)$, van der Waals constant (b), Relaxation strength (r), Relative association $\left(\mathrm{R}_{\mathrm{A}}\right)$ and Ultrasonic attenuation $\left(\alpha / \mathrm{f}^{2}\right)$ of Pyrazoline derivatives with

DMF at $303.15 \mathrm{~K}$.

\begin{tabular}{|c|c|c|c|c|c|}
\hline \multirow{2}{*}{ Concentration } & \multicolumn{5}{|c|}{ Pyrazoline derivatives (P1-P10) } \\
\cline { 2 - 6 } & $\tau$ & $\mathrm{b}$ & $\mathrm{r}$ & $\mathrm{R}_{\mathrm{A}}$ & $\alpha / \mathrm{f}^{2}$ \\
\hline \multirow{7}{*}{$(\mathrm{M})$} & $\left(10^{-10} \mathrm{sec}\right)$ & $\left(\mathrm{m}^{3} /\right.$ mole $)$ & & $\left(10^{-12} \mathrm{sec}\right)$ \\
\hline 0.02 & 6.066 & 0.0838 & 0.1707 & 1.0215 & 8.2097 \\
\hline 0.04 & 6.0031 & 0.0902 & 0.1558 & 1.0360 & 8.0528 \\
\hline 0.06 & 5.9224 & 0.0962 & 0.1443 & 1.0537 & 7.8908 \\
\hline 0.08 & 5.8859 & 0.1023 & 0.1327 & 1.0675 & 7.7896 \\
\hline 0.1 & 5.8665 & 0.1077 & 0.1257 & 1.0874 & 7.7328 \\
\hline \multicolumn{7}{|c|}{ Compound P1 } \\
\hline 0.02 & 5.9961 & 0.0839 & 0.1362 & 1.0143 & 7.9515 \\
\hline 0.04 & 5.948 & 0.0904 & 0.1292 & 1.0304 & 7.8559 \\
\hline 0.06 & 5.9333 & 0.0964 & 0.1269 & 1.0501 & 7.8261 \\
\hline 0.08 & 5.9142 & 0.1038 & 0.1116 & 1.0504 & 7.7336 \\
\hline 0.1 & 5.8478 & 0.1104 & 0.1069 & 1.0605 & 7.6266 \\
\hline \multicolumn{7}{|c|}{ Compound P3 } \\
\hline 0.02 & 6.5596 & 0.0849 & 0.1933 & 1.0264 & 9.0014 \\
\hline 0.04 & 6.5319 & 0.0924 & 0.19 & 1.0432 & 8.9447 \\
\hline 0.06 & 6.5147 & 0.1011 & 0.1821 & 1.0441 & 8.8780 \\
\hline 0.08 & 6.0741 & 0.1083 & 0.1105 & 1.0457 & 7.9375 \\
\hline 0.1 & 6.0577 & 0.1148 & 0.1081 & 1.0665 & 7.9056 \\
\hline
\end{tabular}


Table 8. Relaxation time $(\tau)$, van der Waals constant (b), Relaxation strength (r), Relative association $\left(\mathrm{R}_{\mathrm{A}}\right)$ and Ultrasonic attenuation $\left(\alpha / \mathrm{f}^{2}\right)$ of Pyrazoline derivatives with DMF at $303.15 \mathrm{~K}$.

\begin{tabular}{|c|c|c|c|c|c|}
\hline \multirow{2}{*}{ Concentration } & \multicolumn{5}{|c|}{ Pyrazoline derivatives (P1-P10) } \\
\hline & $\tau$ & $\mathrm{b}$ & $\mathrm{r}$ & $\mathrm{R}_{\mathrm{A}}$ & $\alpha / \mathrm{f}^{2}$ \\
\hline$(\mathrm{M})$ & $\left(10^{-10} \mathrm{sec}\right)$ & $\left(\mathrm{m}^{3} / \mathrm{mole}\right)$ & & & $\left(10^{-12} \mathrm{sec}\right)$ \\
\hline \multicolumn{6}{|c|}{ Compound P4 } \\
\hline 0.02 & 6.2753 & 0.0844 & 0.1627 & 1.0195 & 8.4524 \\
\hline 0.04 & 6.2323 & 0.0913 & 0.1570 & 1.0372 & 8.3659 \\
\hline 0.06 & 6.226 & 0.0996 & 0.1501 & 1.0374 & 8.3235 \\
\hline 0.08 & 6.0438 & 0.1063 & 0.1234 & 1.0478 & 7.9558 \\
\hline 0.1 & 6.0302 & 0.1124 & 0.1164 & 1.0675 & 7.9063 \\
\hline \multicolumn{6}{|c|}{ Compound P5 } \\
\hline 0.02 & 6.2157 & 0.0836 & 0.1967 & 1.0273 & 8.5473 \\
\hline 0.04 & 6.0211 & 0.0895 & 0.1707 & 1.0428 & 8.1490 \\
\hline 0.06 & 5.9707 & 0.0953 & 0.1512 & 1.0590 & 7.9875 \\
\hline 0.08 & 5.9179 & 0.1010 & 0.1374 & 1.0756 & 7.8529 \\
\hline 0.1 & 5.6938 & 0.1065 & 0.1034 & 1.0875 & 7.4110 \\
\hline \multicolumn{6}{|c|}{ Compound P6 } \\
\hline 0.02 & 6.4654 & 0.0831 & 0.2277 & 1.0356 & 9.0678 \\
\hline 0.04 & 6.008 & 0.0888 & 0.1627 & 1.0408 & 8.0923 \\
\hline 0.06 & 5.9383 & 0.0942 & 0.1466 & 1.0576 & 7.9227 \\
\hline 0.08 & 5.9143 & 0.0995 & 0.1432 & 1.0763 & 7.8747 \\
\hline 0.1 & 5.7201 & 0.1047 & 0.1011 & 1.0868 & 7.4354 \\
\hline
\end{tabular}

Table 9. Relaxation time $(\tau)$, van der Waals constant (b), Relaxation strength (r), Relative association $\left(\mathrm{R}_{\mathrm{A}}\right)$ and Ultrasonic attenuation $\left(\alpha / \mathrm{f}^{2}\right)$ of Pyrazoline derivatives with DMF at $303.15 \mathrm{~K}$.

\begin{tabular}{|c|c|c|c|c|c|}
\hline \multirow{2}{*}{ Concentration } & \multicolumn{5}{|c|}{ Pyrazoline derivatives (P1-P10) } \\
\cline { 2 - 6 } & $\tau$ & $\mathrm{b}$ & $\mathrm{r}$ & $\mathrm{R}_{\mathrm{A}}$ & $\alpha / \mathrm{f}^{2}$ \\
\hline \multicolumn{7}{|c|}{$(\mathrm{M})$} & $\left(10^{-10} \mathrm{sec}\right)$ & $\left(\mathrm{m}^{3} / \mathrm{mole}\right)$ & \multicolumn{7}{c|}{ Compound P7 } & $\left(10^{-12} \mathrm{sec}\right)$ \\
\hline 0.02 & 6.5304 & 0.0838 & 0.2012 & 1.0294 & 9.0052 \\
\hline 0.04 & 6.3339 & 0.0902 & 0.1764 & 1.0430 & 8.6018 \\
\hline 0.06 & 6.2071 & 0.0963 & 0.1536 & 1.0585 & 8.3150 \\
\hline 0.08 & 6.1773 & 0.1021 & 0.1374 & 1.0762 & 8.1972 \\
\hline 0.1 & 6.0081 & 0.1078 & 0.1069 & 1.0889 & 7.8356 \\
\hline \multicolumn{7}{|c|}{ Compound P8 } \\
\hline 0.02 & 6.5079 & 0.0837 & 0.2201 & 1.0315 & 9.0821 \\
\hline 0.04 & 6.461 & 0.0900 & 0.2189 & 1.0472 & 9.0102 \\
\hline 0.06 & 6.4443 & 0.0958 & 0.2012 & 1.0637 & 8.8864 \\
\hline 0.08 & 6.4341 & 0.1028 & 0.1956 & 1.0679 & 8.8415 \\
\hline
\end{tabular}




\begin{tabular}{|c|c|c|c|c|c|}
\hline 0.1 & 6.3186 & 0.1082 & 0.1832 & 1.0853 & 8.6167 \\
\hline \multicolumn{5}{|c|}{ Compound P9 } \\
\hline 0.02 & 6.5752 & 0.0834 & 0.2311 & 1.0355 & 9.2415 \\
\hline 0.04 & 6.4477 & 0.0899 & 0.2123 & 1.0447 & 8.9537 \\
\hline 0.06 & 6.2356 & 0.0961 & 0.1832 & 1.0533 & 8.5035 \\
\hline 0.08 & 6.0588 & 0.1026 & 0.1570 & 1.0589 & 8.1330 \\
\hline 0.1 & 6.0455 & 0.1091 & 0.1443 & 1.0655 & 8.0549 \\
\hline \multicolumn{7}{|c|}{ Compound P10 } \\
\hline 0.02 & 6.2396 & 0.0833 & 0.1809 & 1.0299 & 8.4972 \\
\hline 0.04 & 6.0715 & 0.0897 & 0.1524 & 1.042 & 8.1279 \\
\hline 0.06 & 6.0432 & 0.0966 & 0.1489 & 1.0514 & 8.0737 \\
\hline 0.08 & 6.0409 & 0.1031 & 0.1478 & 1.0653 & 8.0651 \\
\hline 0.1 & 6.025 & 0.1100 & 0.1374 & 1.0704 & 7.9951 \\
\hline
\end{tabular}

Table 10. Isothermal compressibility $\left(\beta_{\mathrm{T}}\right)$, Isothermal expansion co-efficient $(\alpha)$, Free volume $\left(\mathrm{V}_{\mathrm{f}}\right)$ and Internal pressure $\left(\pi_{\mathrm{i}}\right)$ of Pyrazoline derivatives with DMF at $303.15 \mathrm{~K}$

\begin{tabular}{|c|c|c|c|c|}
\hline \multirow{2}{*}{ Concentration } & \multicolumn{4}{|c|}{ Pyrazoline derivatives (P1-P10) } \\
\hline & $\beta_{\mathrm{T}}$ & $\alpha$ & $\mathrm{V}_{\mathrm{f}}$ & $\pi_{\mathrm{i}}$ \\
\hline (M) & $\left(10^{-14} \mathrm{~m}^{2} \mathrm{~N}^{-1}\right)$ & $\left(10^{4} \mathrm{~K}^{-1}\right)$ & $\left(10^{-6} \mathrm{~m}^{3} / \mathrm{mole}\right)$ & $\left(10^{6} \mathrm{~Pa}\right)$ \\
\hline \multicolumn{5}{|c|}{ Compound P1 } \\
\hline 0.02 & 0.6694 & 1.0633 & 0.1610 & 4.8309 \\
\hline 0.04 & 0.6429 & 1.0526 & 0.1803 & 4.4289 \\
\hline 0.06 & 0.6182 & 1.0424 & 0.2005 & 4.0963 \\
\hline 0.08 & 0.5976 & 1.0336 & 0.2197 & 3.8133 \\
\hline 0.1 & 0.5774 & 1.0247 & 0.2371 & 3.5921 \\
\hline \multicolumn{5}{|c|}{ Compound P2 } \\
\hline 0.02 & 0.6429 & 1.0527 & 0.1592 & 4.8448 \\
\hline 0.04 & 0.6234 & 1.0446 & 0.1791 & 4.4325 \\
\hline 0.06 & 0.6059 & 1.0372 & 0.1977 & 4.1084 \\
\hline 0.08 & 0.5930 & 1.0316 & 0.2192 & 3.7778 \\
\hline 0.1 & 0.5817 & 1.0266 & 0.2435 & 3.5014 \\
\hline \multicolumn{5}{|c|}{ Compound P3 } \\
\hline 0.02 & 0.6881 & 1.0707 & 0.1491 & 4.9129 \\
\hline 0.04 & 0.6699 & 1.0635 & 0.1698 & 4.4461 \\
\hline 0.06 & 0.6612 & 1.0601 & 0.1937 & 4.0082 \\
\hline 0.08 & 0.5956 & 1.0327 & 0.2241 & 3.6467 \\
\hline 0.1 & 0.5782 & 1.0251 & 0.2448 & 3.4069 \\
\hline
\end{tabular}


Table 11. Isothermal compressibility $\left(\beta_{\mathrm{T}}\right)$, Isothermal expansion co-efficient $(\alpha)$, Free volume $\left(\mathrm{V}_{\mathrm{f}}\right)$ and Internal pressure $\left(\pi_{\mathrm{i}}\right)$ of Pyrazoline derivatives with DMF at $303.15 \mathrm{~K}$.

\begin{tabular}{|c|c|c|c|c|}
\hline \multirow{2}{*}{ Concentration } & \multicolumn{4}{|c|}{ Pyrazoline derivatives (P1-P10) } \\
\hline & $\beta_{\mathrm{T}}$ & $\alpha$ & $\mathrm{V}_{\mathrm{f}}$ & $\pi_{\mathrm{i}}$ \\
\hline$(\mathrm{M})$ & $\left(10^{-14} \mathrm{~m}^{2} \mathrm{~N}^{-1}\right)$ & $\left(10^{4} \mathrm{~K}^{-1}\right)$ & $\left(10^{-6} \mathrm{~m}^{3} / \mathrm{mole}\right)$ & $\left(10^{6} \mathrm{~Pa}\right)$ \\
\hline \multicolumn{5}{|c|}{ Compound P4 } \\
\hline 0.02 & 0.6634 & 1.0609 & 0.1537 & 4.8820 \\
\hline 0.04 & 0.6430 & 1.0527 & 0.1737 & 4.4479 \\
\hline 0.06 & 0.6364 & 1.0500 & 0.1969 & 4.0265 \\
\hline 0.08 & 0.6047 & 1.0366 & 0.2221 & 3.7024 \\
\hline 0.1 & 0.5841 & 1.0277 & 0.2405 & 3.4755 \\
\hline \multicolumn{5}{|c|}{ Compound P5 } \\
\hline 0.02 & 0.6908 & 1.0717 & 0.1584 & 4.8644 \\
\hline 0.04 & 0.6513 & 1.0561 & 0.1799 & 4.4531 \\
\hline 0.06 & 0.6202 & 1.0432 & 0.1967 & 4.1461 \\
\hline 0.08 & 0.5955 & 1.0327 & 0.2146 & 3.8763 \\
\hline 0.1 & 0.5598 & 1.0168 & 0.2392 & 3.6092 \\
\hline \multicolumn{5}{|c|}{ Compound P6 } \\
\hline 0.02 & 0.7172 & 1.0818 & 0.1524 & 4.9480 \\
\hline 0.04 & 0.6453 & 1.0536 & 0.1770 & 4.5026 \\
\hline 0.06 & 0.6172 & 1.0420 & 0.1941 & 4.1968 \\
\hline 0.08 & 0.5999 & 1.0346 & 0.2113 & 3.9339 \\
\hline 0.1 & 0.5585 & 1.0162 & 0.2311 & 3.6929 \\
\hline
\end{tabular}

Table 12. Isothermal compressibility $\left(\beta_{\mathrm{T}}\right)$, Isothermal expansion co-efficient $(\alpha)$, Free volume $\left(\mathrm{V}_{\mathrm{f}}\right)$ and Internal pressure $\left(\pi_{\mathrm{i}}\right)$ of Pyrazoline derivatives with DMF at $303.15 \mathrm{~K}$.

\begin{tabular}{|c|c|c|c|c|}
\hline \multirow{2}{*}{ Concentration } & \multicolumn{4}{|c|}{ Pyrazoline derivatives (P1-P10) } \\
\cline { 2 - 5 } & $\beta_{\mathrm{T}}$ & $\alpha$ & $\mathrm{V}_{\mathrm{f}}$ & $\pi_{\mathrm{i}}$ \\
\hline$(\mathrm{M})$ & $\left(10^{-14} \mathrm{~m}^{2} \mathrm{~N}^{-1}\right)$ & $\left(10^{4} \mathrm{~K}^{-1}\right)$ & $\left(10^{-6} \mathrm{~m}^{3} / \mathrm{mole}\right)$ & $\left(10^{6} \mathrm{~Pa}\right)$ \\
\hline \multicolumn{5}{|c|}{ Compound P7 } \\
\hline 0.02 & 0.6936 & 1.0728 & 0.1483 & 4.9641 \\
\hline 0.04 & 0.6566 & 1.0582 & 0.1696 & 4.5187 \\
\hline 0.06 & 0.6227 & 1.0443 & 0.1888 & 4.1742 \\
\hline 0.08 & 0.5951 & 1.0325 & 0.2046 & 3.9095 \\
\hline 0.1 & 0.5615 & 1.0176 & 0.2256 & 3.6489 \\
\hline \multicolumn{5}{|c|}{ Compound P8 } \\
\hline 0.02 & 0.7123 & 1.0800 & 0.1513 & 4.9375 \\
\hline 0.04 & 0.6968 & 1.0741 & 0.1707 & 4.5165 \\
\hline 0.06 & 0.6639 & 1.0611 & 0.1850 & 4.2181 \\
\hline 0.08 & 0.6548 & 1.0575 & 0.2050 & 3.8891 \\
\hline 0.1 & 0.6290 & 1.0469 & 0.225 & 3.6432 \\
\hline
\end{tabular}




\begin{tabular}{|c|c|c|c|c|}
\hline \multicolumn{5}{|c|}{ Compound P9 } \\
\hline 0.02 & 0.7290 & 1.0832 & 0.1500 & 4.9601 \\
\hline 0.04 & 0.6919 & 1.0721 & 0.1699 & 4.5256 \\
\hline 0.06 & 0.6547 & 1.0574 & 0.1922 & 4.1547 \\
\hline 0.08 & 0.6254 & 1.0454 & 0.2158 & 3.8292 \\
\hline 0.1 & 0.6091 & 1.0385 & 0.2346 & 3.5754 \\
\hline \multicolumn{5}{|c|}{ Compound P10 } \\
\hline 0.02 & 0.6723 & 1.0645 & 0.1545 & 4.9151 \\
\hline 0.04 & 0.6348 & 1.0493 & 0.1754 & 4.4850 \\
\hline 0.06 & 0.6241 & 1.0449 & 0.1968 & 4.1084 \\
\hline 0.08 & 0.6122 & 1.0399 & 0.2165 & 3.8138 \\
\hline 0.1 & 0.5994 & 1.0344 & 0.2374 & 3.5413 \\
\hline
\end{tabular}

\section{RESULTS AND DISCUSSION}

From the Tables (1-3) it is known that, the ultrasonic velocity (U), density $(\rho)$ and viscosity $(\eta)$ increases with increase in concentration. Based on the model for sound propagation proposed by Eyring and Kincaid [23], ultrasonic velocity should increase, if the inter molecular free length decreases and vice verse. The linear variation of density and viscosity indicates that there exist a strong interaction between solute and solvent.

In fact, the molecular association increases ultrasonic velocity $(\mathrm{u})$ and acoustic impedance $(\mathrm{Z})$, decreases intermolecular free length (Lf) and adiabatic compressibility $(\beta)$. A reduction in adiabatic compressibility $(\mathrm{Ks})$ is an indication that component molecules are held close to each other. The decrease in the values of adiabatic compressibility $(\beta)$ and inter molecular free length (Lf) with increase in ultrasonic velocity (u) further strengthens the strong molecular association between the unlike molecules through hydrogen bonding.

Acoustic impedance increases with increase in concentration. Specific acoustic impedance is directly proportional to ultrasonic velocity and inversely proportional to adiabatic compressibility and shows similar behaviour to that of ultrasonic velocity and opposite to that of adiabatic compressibility [24]. Decrease in adiabatic compressibility might be due to aggregation of solvent molecules around solute molecules. Non linear variation of adiabatic compressibility as a function of composition on of liquid mixture is sufficient evidence for existence of molecular interactions in solutions [25-27].

Intermolecular free length decreases with increase in concentration. The decrease in free length is due to the close packing of the molecules inside the shield, which may be brought by strengthening of molecular interactions. This may be attributed to the fact that the intermolecular interactions might have resulted in a decreased intermolecular free length and a compact structural arrangement.

The Relative association decreases with increase in concentration. Decrease in relative association which indicates the breaking up of the solvent molecules on addition of solute [28]. The relaxation time decreases with increase in concentration. The variations in specific relaxation time are mainly due to the change in viscosity of solutions due to both concentration and temperature. The dispersion of the ultrasonic velocity in the system may contain information about the characteristic time $(\tau)$ of the relaxation process that causes dispersion.

Rao's constant and molar compressibility increases with increase in concentration which indicates that the magnitude of interactions is enhanced. The ultrasonic attenuation 
decreases with increase in concentration. The variation of ultrasonic attenuation with increase in concentration is non-linear. This non-linear variation of absorption with concentration of one component strongly supports the presence of strong inter molecular interaction [29]. The van der Waals constant increases with increase in concentration and the Isothermal compressibility decreases with increase in concentration of the solute.

Internal pressure in a liquid system is a measure of intermolecular cohesive forces. The internal pressure decreases with increase in concentration of solute, which indicates the decrease in cohesive forces. This suggests close packing of the molecules inside the shield, which may be brought about by the increasing magnitude of interactions [30-31].

The free volume increases with increase in concentration. The decrease in molecular association of solvent molecule causes an increase in free volume. This may be explained that there is a tendency for the solute molecules to move away from each other, reducing the possibility for interaction, which may further reduce the cohesive force and ultimately lead to an increase in free volume [32].

\section{INTERACTIONS IN PYRAZOLINE - DIMETHYLFORMAMIDE}

Dimethylformamide is polar in nature. In general, the $-\mathrm{OH}$ group is of particular interest because of its highly polar in nature. The associative hydroxyl group in Pyrazoline compound act as proton donor enabling hydrogen bonding with dimethylformamide.

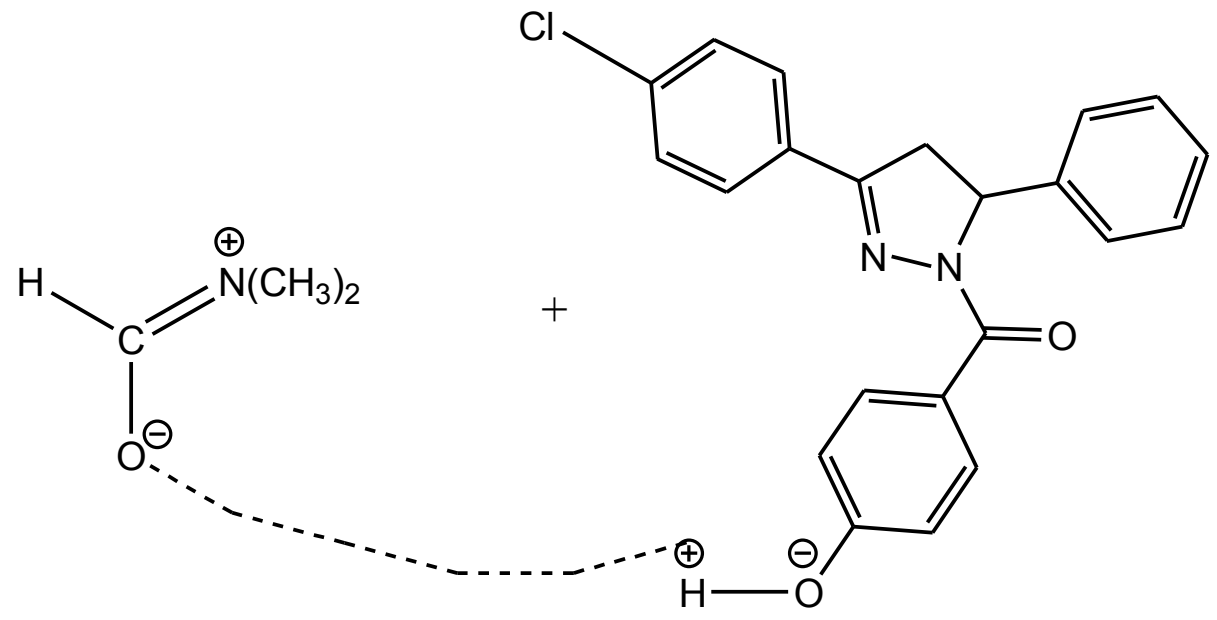

In the above system studied, there is possibility for three types of interactions. (i) Intermolecular hydrogen bonding takes place between carbonyl oxygen of dimethylformamide with hydroxyl hydrogen of pyrazoline compound. (ii) a dipole-dipole interaction takes place between carbonyl carbon of dimethylformamide and hydroxyl oxygen of Pyrazoline compound (iii) an electron donor - electron acceptor complex formation takes between the electron donating methyl group of dimethylformamide and the electron withdrawing chlorine atom of Pyrazoline compound.

So there is existence of solute-solvent interactions between the solvent (dimethylformamide) and solute (pyrazoline compound). 


\section{CONCLUSION}

The acoustical parameters were calculated from the measured ultrasonic velocity, density and viscosity. The existence of molecular interactions in solute-solvent is favoured in the system, which is confirmed by the Complex formations, formation of hydrogen bond, dipole - dipole interactions in solutions and their effect in physical properties of the mixture. Hence it is concluded that there exist a strong molecular interaction between the solute (Pyrazoline derivatives) and the solvent (DMF).

\section{References}

[1] Ali A., Nain A. K., Indian J Phys. 74B (2000) 63.

[2] Kannappan V., Jaya Santhi R., J Acous Soc Ind. 29 (2001) 192.

[3] Rastogi M., Awasthi A., Gupta M., Shukla J. P., Indian J Pure Appl Phys. 40 (2002) 256.

[4] Nain A. K., Phys Chem Liq. 45 (2007) 371.

[5] Pal A., Das G., J. Pure \& Applied Ultrasonic 21 (1990) 9.

[6] Tabhane V. A., Patki B. A., Indian J. Pure \& Applied Physics 23 (1985) 58.

[7] Bhandakkar V. D., Adv. Applied Sci. Res 2(3) (2011) 198-207.

[8] George J., Sastry N. V., Patel S. R., Valand M. K., J. Chem. Eng. 47 (2002) 262.

[9] Bhandakkar V. D., Tabhane V. A., Ghosh S., Indian J. Pure \& Applied Physics 41 (2003) 849-854.

[10] S. Prakash, J. Singh, S. Srivastava, Acoustica 65 (1988) 263.

[11] C. Chemarayappa, K. Ramababu, P. Venkateswaralu, G. K. Raman, Acoustics Lett. 15 (1991) 83.

[12] C. Padrnasree, K. Ravindraprasad, Indian J. Pure Appl. Phys. 32 (1994) 954.

[13] Nain AK, et al., Journal of Fluid Phase Equilibria 265(1-2) (2008) 46-56.

[14] Bhoj Bhadur Gurung, Mahendra Nath Roy, Journal of Solution Chemistry 35 (2006) 1587-1606.

[15] Zareena Begaum, et al., Journal of Molecular Liquids 178 (2013) 99-112

[16] Thanuja B, Charles Kanagam, et al., Journal of Ultrasonics Sonochemistry 18 (2011) $1274-1278$

[17] Manohar Murthi, N. Nagbhushnam, Indian J Chem. 23 (1984) 510.

[18] Khasare S. B., Indian J Pure \& Appl Phys. 25 (1987) 182.

[19] Rajgopal K., Chenthilnath S., Journal of Chemical Engineering 18 (2010) 806-816.

[20] Anil Kumar Nain, Journal of Fluid Phase Equilibria 259(2) (20070 218-227.

[21] Shahla Parveen, Divya Shukla, et al., Journal of Applied Acoustics 70(3) (2009) 507513. 
[22] Rajgopal K, Chenthilnath S., Journal of Molecular Liquids 160(2) (2011) 72-80.

[23] Eyring H., Kincaid J. F., J. Chem. Phys. 6 (1938) 620-629.

[24] Padma S., There., Rasayan J. Chem 6(2) (2013) 111.

[25] Fort R. J., More W. H., Trans. Faraday Soc. 61 (1965) 2102.

[26] Baluja S., Oza S., Fluid Phase Equil bria 20 (20 2) 1.

[27] Oswal S. L., Patel I. N., Fluid Phase Equil bria, 149 (1998)

[28] Shashikant et al., Journal of Chemical and Pharmaceutical Research 4(8) (2012) 33791.

[29] J. L. Hunter, D. Dossa, J. Haus, J. Chem. Phys. 60 (1974) 4605.

[30] Govindarajan S., Venu Kanapan, Naresh M. D., Venkatabopathy K., Lokanadam B., J. Mol. Liqs. 107(1-3) (2003) 289-316.

International Research Journal of Pure \& Aplied Chemistry 4(2) (2014) 213-226.

[31] Ali A, Abida Hyder S, Nain AK., Indian J Phy. 76B(15) (2002).

[32] Jayakumar S., et al., J. Acoust. Soc., Ind. 30 (2002) 84. 